ready to commence and soon received the go-ahead. The pace was quickening again.

The Italian general election, set for June 20 , just two days after the crucial Council of Research Ministers meeting, now intervened. With the prospect of an upset result producing an Italian government which might disagree with any decision taken by the Council, it began to seem pointless having the meeting at all. At Italy's request it was decided that the meeting would not take place. The Dutch proposed a meeting for mid-July; this was agreed in principle. As late as the first week of June, Brunner was expressing the hope that a decision on the site would be taken at such a meeting. Days later it was clear that no meeting was likely before the summer recesss. No early decision on the site would therefore be possible unless the idea of a determination by the nine Foreign Ministers was resuscitated.

The Commission had itself come round to this view. It had also been busy on the central problem of the site: by the end of May it had finished preparing additional information for the Council of Research Ministers in order to make the choice easier. This included, first of all, the news that consultations concerning the higher cost estimates had produced agreement that the extra amounts involved were in fact negligible, at about 5 mua. The Commission also contended that insufficient emphasis had been attached previously to the matter of the power supply: if JET was to be fed directly, Ispra, fed by a line from a thermal and a hydroelectric station, possessed another advantage over other sites. Finally, the Commission stressed the costs of delay. Increasingly frustrated, it therefore argued that no attempt to justify a further delay could be based on arguments of a scientific, technical, financial or adminstrative nature.

With no prospect of a meeting of the Council of Research Ministers before mid-October, and the need for decisions growing more desperate, it was now time to change tack. At the end of June, therefore, and at the request of the Dutch (who were about to take over the Council presidencies), the nine Foreign Ministers had a brief exchange on the siting of JET. The Commission was now looking for a decision of principle on the building of JET before the recess in order at least to supply more adhesive to the JET team than the second six-month extension to contracts just put together.

The matter was put on the agenda of the Heads of Government meeting on July 12. The boost they were busy giving to the Community did not extend as far as JET, however, and it was not discussed. Assurances were

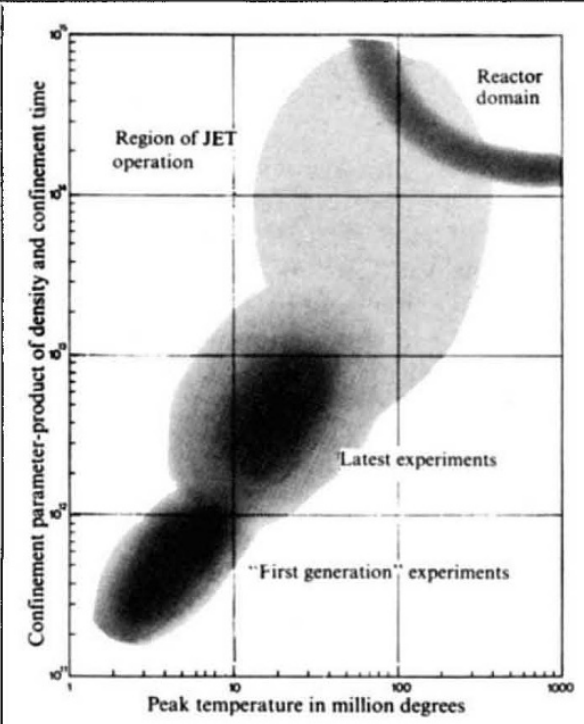

\section{Fusion research: where it's at}

FusIon research, which seeks to harness the energy released when nuclei of light elements are brought together to form heavier ones, aims ultimately to design a practical fusion reactor that produces electricity economically. The aim is thus to approach more closely the conditions at which the energy released from the deuterium-tritium reaction (the fusion reaction selected as the most promising of several possibilities) is greater than that expended both in heating the plasma in which the reaction occurs, and in losses.

The immediate goals are therefore to find ways of heating the plasma to the necessary temperature, and to confine that plasma for the necessary time. Apart from the passage of electric current, methods of heating currently being investigated include the use of intense laser beams and the use of beams of high-energy neutralised particles originally produced from an ion source. The method of confinement in a doughnut (torus) shape uses a helical magnetic field. This is made up of two components, a toroidal field produced by large external coils, and a poloidal field which, in the case of tokamaks like jet, is produced by a toroidal current in the plasma.

Tokamaks, however, represent only one of three classes of toroidal device now being examined for their confinement capabilities. These are in fact distinguished by the way the poloidal field is produced:

(a) Tokamaks, and Reversed-field Pinches (high beta systems). In tokamaks the toroidal field is greater than the poloidal field; in reversed-field pinches the two are about the same and the toroidal field is reversed in the outer

given that last week's Foreign Ministers meeting would deal with the matter in some detail though it was not on the official agenda. Nothing approaching progress which would satisfy the Commission resulted. The recess is now imminent. October is two more months away. regions of the plasma. In both, the plasma current which heats the plasma also creates the poloidal field.

(b) Stellarators (low beta systems). In this class of device, currents in helical conductors wrapped round the torus and outside the plasma produce the field which, with the toroidal field, confines the plasma.

(c) Toroidal multipole. Here the poloidal field is produced by a steady current flowing in a levitated superconducting ring located in the centre of the vacuum chamber with the plasma.

Magnetic confinement produces a plasma of modest density, so the confinement time necessary to boost the "confinement parameter" (density x confinement time) is longer compared to a plasma of very high density.

Obtaining a high-density plasma is the aim of inertial confinement, in which the necessary time of confinement is actually shorter than the period in which the particles will escape from the reacting zone. This confinement is achieved by irradiation with high-power laser beams. Extremely intense, short laser pulses bombard a solid deuteriumtritium fuel pellet, causing it to turn into a plasma so hot and so compressed that fusion reactions yield a surplus of energy before the pellet decays as a result of expansion. The method does not simply offer the possibility of avoiding the difficulties of using magnetic fields for confinement; it also offers the theoretical possibility of using a series of laser pulses to cause a succession of these explosions.

The selling of fusion power has turned largely on its ostensible advantages in respect of the fuel it uses and its safety. But the supply of lithium (used to produce tritium) is at least not certain, and neutron production in the reactor has the potential to make the reactor materials highly radioactive. There are other problems relating to possible lithium fires and tritium leakage. And since fusion is so patently a prospect for the distant future, guesses about its economics remain exactly that--all of which makes any choice between fusion and fast-breeder fission, if such a choice exists, correspondingly more difficult to make.

JET is effectively a "third generation" tokamak device. Its equivalent in the USSR is known as T-20, in the USA as TFTR, and in Japan as JT 60. "Second generation" tokamaks include DITE at Culham, T-10 in the USSR and PLT in the USA.

For the post-Jet period, the following sort of sequence is imagined:

1980-1985 Tritium burning experiment

1985-1990 Experimental reactor

1990-2000 Prototype

2000-2010 Demonstration

Other common facilities envisaged over this period include a material testing facility and a superconducting magnet assembly.

\section{JET lag importance}

The persistent procrastination, far from indicating that the EEC perceives the JET decision as having comparatively minor consequence, is precisely a reflection of the project's importance. JET is a major Community research project, perhaps the first of its type, 